\title{
Bilateral Optic Neuritis Associated with Central Nervous System Infection in an Adult
}

Jin Young Seo, MD (1); Hong-Ki Song, MD, PhD (1); Ju-Hun Lee, MD, PhD (D; Jong Seok Bae, MD, PhD (D; Yerim Kim, MD, MSc $\mathbb{D}$

Department of Neurology, Kangdong Sacred Heart Hospital, Hallym University College of Medicine, Seoul, Korea

We present a case report of bilateral optic neuritis related to meningoencephalitis in an adult. A 62-year-old woman was admitted with sudden bilateral visual loss, headache, and fever. Neurological examination showed no specific findings except light perception on bilateral visual acuities. Cerebrospinal fluid examination and brain magnetic resonance imaging indicated meningoencephalitis and infectious optic neuropathy. She was treated with intravenous methylprednisolone and acyclovir for the infectious optic neuritis. After treatment, her vision improved, such that she could read text up close. Although the infectious source was not confirmed, the imaging test results and clinical symptoms strongly indicated that infection did occur. In conclusion, it is very important for clinicians to perform the necessary tests and initiate appropriate treatment as soon as possible for patients with visual impairment of unknown cause.

J Neurosonol Neuroimag 2020;12(1):40-43

Key Words: Optic neuritis; Meningoencephalitis; Vision disorders; Visual acuity
Received: September 14, 2019

Revised: October 8, 2019

Accepted: October 10, 2019

Correspondence:

Yerim Kim, MD, MSc

Department of Neurology,

Kangdong Sacred Heart Hospi-

tal, Hallym University College

of Medicine, 150 Seongan-ro,

Gangdong-gu, Seoul 05355,

Korea

Tel: $+82-2-2224-2206$

Fax: +82-2-2224-2339

E-mail: brainyrk@gmail.com
Optic neuritis is defined as inflammation that damages the optic nerve. Common symptoms include eye pain and temporary vision loss. Optic neuritis is often idiopathic and can occur in association with demyelinating diseases, other rare autoimmune diseases, infectious diseases, and inflammatory and immune responses to vaccination. ${ }^{1}$ In adults, optic neuritis is mainly associated with autoimmune diseases such as multiple sclerosis and typically occurs in one eye. In contrast, post-infectious optic neuritis is common in children and mainly involves both eyes. ${ }^{2}$

Bilateral optic neuritis associated with central nervous system (CNS) infection is rare in adults. Only a few cases of optic neuritis post-encephalitis have been reported in the recent years. ${ }^{3,4}$ Cases of optic neuritis accompanying encephalitis and meningitis are even more rare. We would like to present a case report of bilateral optic neuritis, which is suspected to be related to CNS infection in an adult.

\section{CASE}

A 62-year-old woman was admitted with sudden bilateral visual loss since 3 days. She had also had headache, dizziness and fever for 4 days. She was receiving medication for hypertension and had no history of going abroad.

On physical examination, she had a mild fever of $37.8^{\circ} \mathrm{C}$. Neurological examination showed no specific findings involving the motor and sensory systems. Meningeal irritation sign was negative, and all functions of cranial nerves were normal. On ophthalmologic examination, she retained some light perception on bilateral visual acuities. The pupils were equal, round, and reactive to light. A relative afferent pupillary defect was not definite in both eyes. Furthermore, there was no limitation or pain on eye movement. Dilated fundus evaluation revealed the presence of blurred disc margins, which led to a suspicion of papilledema (Fig. 1A).

Routine blood chemistry showed normal results. 
Additional laboratory tests including those for fluorescent antinuclear antibody (FANA), perinuclear antineutrophil cytoplasmic antibody (p-ANCA), cytoplasmic antineutrophil cytoplasmic antibody (c-ANCA) anti-cardiolipin antibody, anti-beta-2 glucose 6 phosphate isomerase (GPI) antibody, vitamin B1, B6, B12, folate, anti-aquaporin 4 antibody, and paraneoplastic autoantibody were also showed normal results. Tests involving $\mathrm{N}$-methyl-D-aspartate receptor (NMDAR), voltage gated potassium channels, and $\alpha$-amino-3-hydroxy-5-methyl-4-isoxazolepropionic acid (AMPA) were not performed because of the lack of encephalitis characteristics accompanying the optic neuritis. In contrast, thyroid function tests showed a decreased level of thyroid stimulating hormone of $0.223 \mathrm{uIU} / \mathrm{mL}$, increased anti-microsome antibody level of more than 1,300 U/ $\mathrm{mL}$, and increased anti-thyroglobulin antibody level of 83.8 U/mL. However, triiodothyronine $\left(\mathrm{T}_{3}\right)$ and free thyroxine $\left(\mathrm{T}_{4}\right)$ levels were within the normal range.

The results showed delayed P1oo latencies in full field visual evoked potential (VEP) bilaterally. These findings were indicative of prechiasmatic conduction defect in bilateral VEP pathways. Cerebrospinal fluid (CSF) examination was performed: CSF opening pressure, $15 \mathrm{~cm}$ $\mathrm{H}_{2} \mathrm{O}$; white blood cell (WBC) count, $140 / \mathrm{mm}^{3}$ (79\% lymphocytes); protein, $175.0 \mathrm{mg} / \mathrm{dL}$; and glucose, $64 \mathrm{mg} / \mathrm{dL}$ (serum glucose: $115 \mathrm{mg} / \mathrm{dL}$ ). There was no viable cell on CSF cytology. No oligoclonal band was detected in the CSF. Brain magnetic resonance imaging (MRI) showed diffuse swelling and heterogeneous enhancement in both optic nerves, which suggested infectious optic neuropathy (Fig. 2A). In addition, diffuse leptomeningeal enhancement and multiple hyperintensity lesions indicated meningitis and encephalitis (Fig. 2B). However, none were confirmed in the viral polymerase chain reaction (PCR), including herpes simplex virus types 1 and 2, Epstein-Barr virus, Enterovirus, cytomegalovirus culture, and varicella zoster virus culture. In addition, antibody-based screening test for tuberculosis, parasites, fugal, and bacterial infections, including Japanese encephalitis and Lyme disease, showed negative results.

She was treated with intravenous methylprednis-
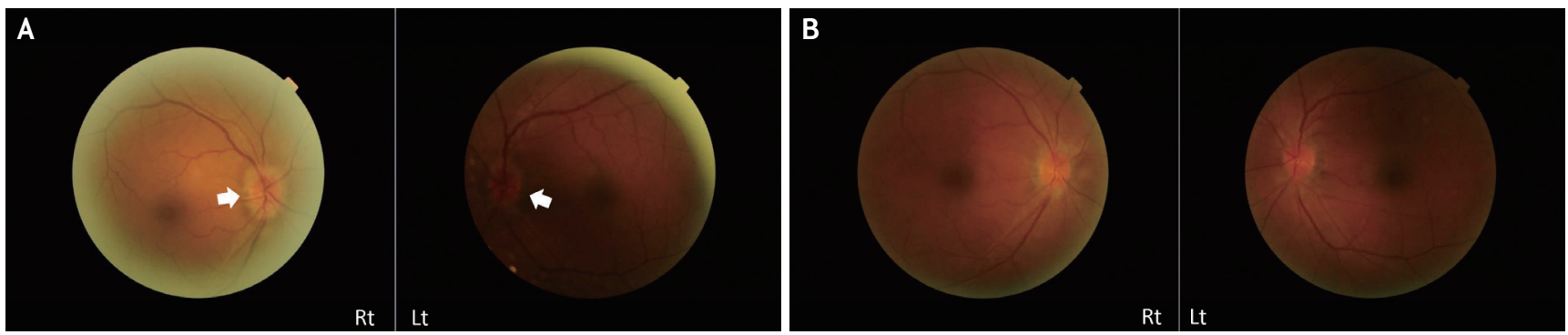

FIG. 1. Fundoscopy shows (A) bilateral disc margin (white arrow) blurring and papilledema, (B) improved papilledema.
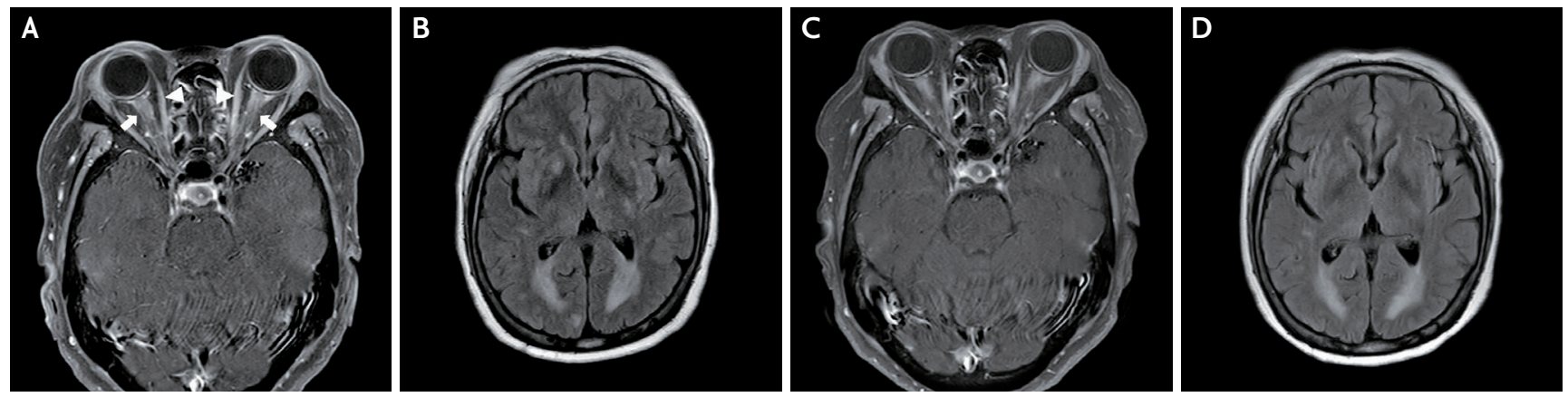

FIG. 2. Brain fat suppressed magnetic resonance imaging (MRI) shows (A) diffuse swelling and heterogeneous enhancement in both optic nerves (white arrows) without swelling of medial rectus muscle (white arrowheads). (B) Brain MRI fluid attenuation inversion recovery (FLAIR) sequence shows multiple hyperintense lesions on both basal ganglia, thalamus, left internal capsule. (C) Diffuse swelling and heterogeneous enhancement in both optic nerves improved after 9 days of treatment in brain fat suppressed MRI, and (D) decreased size of hyperintense lesions in brain MRI FLAIR sequence. 
olone and acyclovir for infectious optic neuritis and meningoencephalitis. After 9 days of treatment, brain MRI showed improved meningoencephalitis and optic neuritis (Fig. 2C, D). Follow-up CSF findings were as follows: opening pressure $15 \mathrm{cmH}_{2} \mathrm{O}, \mathrm{WBC} 100 / \mathrm{mm}^{3}$ (95\% lymphocytes), protein $109.0 \mathrm{mg} / \mathrm{dL}$, and glucose $77 \mathrm{mg} / \mathrm{dL}$ (serum glucose $248 \mathrm{mg} / \mathrm{dL}$ ). CSF cytology showed few macrophages. Two weeks after treatment, her vision improved, and she was able to read text; the bilateral papilledema also improved (Fig. 1B). Two months later, her vision was intact, and the disc swelling in both eyes also improved.

\section{DISCUSSION}

There are various possible causes of spontaneous bilateral optic neuritis. First, optic neuritis is often a symptom of demyelinating diseases such as multiple sclerosis or neuromyelitis optica spectrum disorders (NMOSD). ${ }^{2}$ In this case, brain MRI findings were consistent with meningoencephalitis. No spinal cord abnormalities were found on spine MRI. In addition, tests for the oligoclonal band of CSF and anti-aquaporin 4 antibody were performed twice to exclude multiple sclerosis and NMOSD but showed negative results. Second, the possibility of other autoimmune diseases should be considered. Thyroid function tests revealed subclinical hyperthyroidism, ${ }^{5}$ including elevated antimicrosome antibody and anti-thyroglobulin levels. When optic neuropathy is associated with thyroid dysfunction, it is called dysthyroid optic neuropathy (DON). DON is mainly related to Graves' disease, and its mechanism is explained by compression of the optic nerve caused by extraocular muscle enlargement. ${ }^{6}$ In this case, it is difficult to consider DON because the swelling of the medial rectus muscle was not confirmed in the brain MRI (Fig. 2A). In addition, although there are studies showing the relationship between antithyroid antibodies and demyelinating diseases, ${ }^{7}$ we have previously explained that this case is less likely to be associated with any demyelinating disease. Third, optic neuritis due to infectious disease or inflammatory reaction should be considered. Optic disc swelling on fundoscopy and the swelling and enhancement of the optic nerve on brain MRI suggest papillitis, which can be caused by almost all infections. ${ }^{8}$ Although there is no confirmed infectious source, it is most likely that the diagnosis is infectious optic neuritis accompanying meningoencephalitis, when considering the imaging test results, CSF findings, and clinical symptoms.

Since it is clinically difficult to identify the cause of atypical optic neuritis, clinicians should try to perform active screening and appropriate treatment. Intravenous corticosteroids have been effective in most optic neuritis patients. ${ }^{1-4,8}$ When infectious optic neuropathy is suspected, as in this case, it is helpful to use antiviral drugs and steroids with caution. ${ }^{8}$ In conclusion, it is very important for the clinician to perform the necessary tests and initiate appropriate treatment as soon as possible for patients with visual impairment of unknown cause.

\section{Acknowledgments}

This research was supported by the Basic Science Research Program through the National Research Foundation of Korea (NRF) funded by the Ministry of Science and ICT (NRF-2018R1C1B5086320).

\section{Conflicts of Interest}

No potential conflicts of interest relevant to this article was reported.

\section{REFERENCES}

1. Hoorbakht H, Bagherkashi F. Optic neuritis, its differential diagnosis and management. Open Ophthalmol J. 2012;6:6572.

2. de la Cruz J, Kupersmith MJ. Clinical profile of simultaneous bilateral optic neuritis in adults. $\mathrm{Br} \mathrm{J}$ Ophthalmol. 2006;90:551-554.

3. Kumar R, Bhargava A, Jaiswal G, Soni VR, Katbamna B, Vashisht A. A case of post encephalitic optic neuritis: clinical spectrum, differential diagnosis and management. J Ophthalmic Vis Res. 2018;13:191-194.

4. Hasan S, Basri HB, Hin LP, Stanslas J. Encephalitis followed by optic neuritis: a case report and review of literature. Pak J Med Sci. 2013;29:859-862.

5. Garg A, Vanderpump MP. Subclinical thyroid disease. $\mathrm{Br}$ Med Bull. 2013;107:101-116.

6. Blandford AD, Zhang D, Chundury RV, Perry JD. Dysthy- 
roid optic neuropathy: update on pathogenesis, diagnosis, and management. Expert Rev Ophthalmol. 2017;12:111-121.

7. Long Y, Zheng Y, Chen M, Zhang B, Gao C, Shan F, et al. Serum thyroid-stimulating hormone and anti-thyroglobulin antibody are independently associated with lesions in spi- nal cord in central nervous system demyelinating diseases. PLoS One. 2014;9:e100672.

8. Kahloun R, Abroug N, Ksiaa I, Mahmoud A, Zeghidi H, Zaouali S, et al. Infectious optic neuropathies: a clinical update. Eye Brain. 2015;7:59-81. 